\title{
Robust Fuzzy Control for Anaerobic Digestion System Subject to Unknown Inputs
}

\author{
Mouna Boughamsa and Messaoud Ramdani
}

\begin{abstract}
Biological processes are complex, nonlinear uncertain systems and the state variables are not all available for measurement. For that, fuzzy modeling and state estimations are powerful methods for the control and diagnosis of these kind of systems. In this paper, we present some results obtained from the design of fuzzy controller and fuzzy sliding mode observer for an anaerobic digestion system.
\end{abstract}

Index Terms-Anaerobic digester, fuzzy controller, linear matrix inequalities, robust state estimation, T-S fuzzy modeling.

\section{INTRODUCTION}

Biological processes are complex, nonlinear and uncertain systems. They are submitted to unexpected perturbations and changes, and in most of the time badly definite. This can cause damages and catastrophic situations. A way to achieve that is by means of robust control system design.

Often, it is more useful to represent nonlinear complex systems by the aggregation of local linear time invariant (LTI) models obtained by linearization around multiple operating points. Fuzzy systems have the capacity to handle in the same framework numeric and linguistic information. This characteristic made these systems very useful to handle expert control tasks [1].

Takagi-Sugeno modeling is always proving the effectiveness to represent nonlinear systems because they permit to represent most of nonlinear systems, whatever is its complexity, by a simple "IF...THEN" structure. The fuzzy representation introduced in [2] constitutes an interesting alternative in the domain of control, observation and diagnosis of nonlinear systems.

Wastewater treatment is an important research field discussed in a lot of publications, because wastewater treatment plants (WWTP) contribution is reducing the impact of human waste [3], [4]. This is achieved by removing most of the oxygen demand caused by chemical and organic wastes in the wastewater prior to its return to the environment.

The anaerobic digestion process is the one waste treatment process which presents a number of advantages. It allows for the stabilization of the waste through the controlled destruction of the organic material to produce an end product (digestate) that can be used as a fertilizer or soil conditioner and it represent a renewable energy source.

Manuscript received June 6, 2013; revised Febraury 27, 2014.

Mouna Boughamsa is with Laboratory of Automatic and Signas Annaba (LASA), Badji-Mokhtar University, Department of Electronic, Annaba, Algeria. (e-mail: boughamsamouna@yahoo.fr).

Messaoud Ramdani is with the Department of Electronics, Faculty of Engineering, University Badji-Mokhtar of Annaba, Algeria. (e-mail: messaoud.ramdani@univ-annaba.dz)
The process produces some energy (biogas), composed of methane and carbon dioxide. This biogas especially methane can be used directly as fuel made from living things (cooking fuel, heating, vehicle fuel, electricity generation) where can helps to replace fossil fuels.

Anaerobic digestion variables are not all available for measurement because hardware sensor measurements does not exist which are very important for control and diagnosis [5]. In order to solve such problem, the use of soft sensors is necessary because they have a lot of advantages (less expensive and easy to implement) in comparison with hard sensors. For that, a fuzzy sliding mode observer (FSMO) is used as soft sensor as shown in a previous paper [6]. Fuzzy controller and observer can be independently designed.

The big challenge of control theory is the use of physical model which are nonlinear and complex. Advanced research is devoted to model-based fuzzy control methods that can guarantee stability and robustness of the closed-loop system [7]-[10].

In this work, we used a fuzzy robust controller for (ADP) described by T-S fuzzy models. An LMI approach has been investigated in many research works [11]-[14] to find the fuzzy controller gains used to construct the state feedback law which guarantee of both the stability and the robustness of the closed loop [15].

In this paper, we investigate the use of T-S fuzzy controller based on robust state estimation obtained by fuzzy sliding mode observer for anaerobic digestion system. The rest of this paper is organized as follows: Section II outlines the fuzzy modeling and Gustafson-Kessel algorithm. Section III is devoted to the T-S fuzzy controller design and the stability of this controller is formulated in terms of linear matrix inequality (LMI) and solved. Section IV describes briefly the analytic anaerobic digestion model. Experimental results are presented in Section V. Finally, some concluding remarks as well as some possible improvements are given in Section VI.

\section{T-S FuZZY MODELING}

It is more useful to represent nonlinear systems (1) by local models (3) obtained by a multiple operating point linearization as shown in previous work [6].

\section{A. T-S Fuzzy model}

Fuzzy models are able to represent complicated strong nonlinear systems by an intelligent system representation. As shown in previous work [6].

$$
\left\{\begin{array}{l}
\dot{x}(t)=f(x, u) \\
y(t)=h(x, u)
\end{array}\right.
$$

The model of the plant is assumed to be given by the T-S models, where the $i$-th rule is of the form: 
Rule $i$ : if $\xi_{1}$ is $M_{1 i}$, and,..., and, $\xi_{g}$ is $M_{g i}$

Then $\left\{\begin{array}{l}\dot{x}(t)=A_{i} x(t)+B_{i} u(t)+E_{i} d(t)+\mathrm{a}_{i} \\ y(t)=C_{i} x(t)+c_{i}\end{array}\right.$

where $x \in R^{n}$ is the state vector, $u \in R^{m}$ is the control input vector, $y \in R^{p}$ is the system output vector and $d \in R^{q}$ contains unknown inputs and it is assumed to be bounded (2) $\xi(t)=\left[\xi_{1}(t), \ldots, \xi_{g}(t)\right]^{\mathrm{T}}$ is the decision vector, $M_{g i}$ are membership functions, $A_{i} \in R^{n \times n}, B_{i} \in R^{n \times m}$, $C_{i} \in R^{p \times n}, E_{i} \in R^{n \times q}$ are known matrices and $i=\{1, \ldots, r\}$ with $r$ is the number of rules.

$$
\|d(t)\| \leq \rho
$$

The whole dynamic system defined by T-S fuzzy system is given by:

$$
\left\{\begin{array}{l}
\dot{x}(t)=\sum_{i=1}^{r} h_{i}(\xi(t))\left[A_{i} x(t)+B_{i} u(t)+E_{i} d(t)+a_{i}\right] \\
y(t)=\sum_{i=1}^{r} h_{i}(\xi(t))\left[C_{i} x(t)+c_{i}\right]
\end{array}\right.
$$

where

$$
\begin{gathered}
h_{i}(\xi(t))=\frac{\mu_{i}(\xi(t))}{\sum_{i=1}^{r} \mu_{i}(\xi(t))} \\
\mu_{i}(\xi(t))=\prod_{j=1}^{g} \mu_{i j}\left(\xi_{j}(t)\right)
\end{gathered}
$$

is the degree of fulfillment of the $i$-th rule, $\mu_{i j}\left(\xi_{j}(t)\right)$ is the grade of membership function of $\xi_{j}(t)$ in $M_{i j}$, and $M_{i j}$ is a fuzzy sets. $h_{i}(\xi(t))$ must satisfy the following constraints:

$$
\left\{\begin{array}{l}
\sum_{i=1}^{r} h_{i}(\xi(t))=1, \\
h_{i}(\xi(t)) \in[0,1], \forall i=1, \ldots, r
\end{array}\right.
$$

In order to obtain $\mathrm{T}-\mathrm{S}$ fuzzy system with a minimum number of rules based on dataset. Gustafson-Kessel (GK) clustering algorithm [16] is used to discover the potential regions of the rules or the operating regimes. For a finite number of clusters each iteration is based on minimizing an objective function that represents the distance from any given data point to a cluster center weighted by that data point's membership grade.

The GK clustering algorithm:

Given $\phi=[x, y]^{T}(1<K<N, m>1)$. The data set to be clustered, where $K$ is the number of clusters, $N$ is the number of input-output simples and $\mathrm{m}$ is a constant (normally chosen to be 2).

Initialize $U^{0}$

For: $l=1, \ldots, m$

1) Compute cluster means:

$$
v_{i}^{(l)}=\frac{\sum_{k=1}^{N}\left[h_{i k}^{(l-1)}\right]^{m} z_{k}}{\sum_{k=1}^{N}\left[h_{i k}^{(l-1)}\right]^{m}}
$$

$i=1, \ldots, K$

2) Compute covariance matrices of $i^{\text {th }}$ cluster:

$$
F_{i}=\frac{\sum_{k=1}^{N}\left[h_{i k}^{(l-1)}\right]^{m}\left[z_{k}-v_{i}^{(l)}\right]\left[z_{k}-v_{i}^{(l)}\right]^{T}}{\sum_{k=1}^{N}\left[h_{i k}^{(l-1)}\right]^{m}}
$$

$k=1, \ldots, N$

3) Compute distances between each cluster:

$$
D_{i k}^{2}=\left[z_{k}-v_{i}^{(l)}\right]^{T}\left[\operatorname{det}\left(F_{i}\right)^{1 / r} F_{i}^{-1}\right]\left[z_{k}-v_{i}^{(l)}\right]
$$

4) Update partition matrix:

If $D_{i k}>0$ for $1 \leq i \leq K, 1 \leq k \leq N$

$$
h_{i k}^{(l)}=\frac{1}{\sum_{j=1}^{K}\left(D_{i k} / D_{j k}\right)^{2 /(m-1)}}
$$

Otherwise $h_{i k}^{(l)}=0$ if $D_{i k}=0$, and $h_{i k}^{(l)} \in[0,1]$. With $\sum_{i=1}^{K} h_{i k}^{(l)}=1$ until $\left\|U^{(l)}-U^{(l-1)}\right\|<\varepsilon$

\section{T-S FUZZY MODEL CONTROL DESIGN}

Fuzzy controller based on robust state estimation using FSMO is designed for each local linear model and shares the same fuzzy sets with the fuzzy model in the premise parts [11].

Rule $i$ :

If $\xi_{1}$ is $M_{1 i}$, and,$\ldots$, and, $\xi_{g}$ is $M_{g i}$

Then $u_{i}(t)=K_{i} x(t)$

Each of the rules can be viewed as describing a local state feedback controller associated with the corresponding local sub-model of the system to be controlled.

The resulting total control action is given by:

$$
u(t)=\sum_{i=1}^{r} h_{i}(\xi(t)) K_{i} x(t)
$$

where $K_{i} \in R^{m \times n}$ is the local feedback gain. Note that the resulting fuzzy controller (9) is nonlinear in general since the coefficient of the controller depends nonlinearly on decision variable vector $\xi(t)$ (a subset of the system input and output).

The closed-loop fuzzy controlled system (FCS) can be represented as

$$
\dot{x}(t)=\sum_{i=1}^{r} \sum_{j=1}^{r} h_{i}(\xi(t)) h_{j}(\xi(t))\left(\mathrm{A}_{i}+\mathrm{B}_{i} \mathrm{~K}_{j}\right) x(t)
$$

Considering the quadratic Lyapunov function [8]:

$$
V(x)=x^{T} P x
$$

Its time derivative $\dot{V}(x)$ is 


$$
\begin{aligned}
\dot{V}(x) & =x^{T}\left(\sum_{i=1}^{r} \sum_{j=1}^{r} h_{i}(\xi) h_{j}(\xi)\left[\left(\mathrm{A}_{i}+\mathrm{B}_{i} \mathrm{~K}_{j}\right)^{T} P+P\left(A_{i}+B_{i} K_{j}\right)\right]\right) x \\
& =\sum_{i=1}^{r} \sum_{j=1}^{r} h_{i}(\xi) h_{j}(\xi) x^{T}\left(\left(\mathrm{~A}_{i}+\mathrm{B}_{i} \mathrm{~K}_{j}\right)^{T} P+P\left(A_{i}+B_{i} K_{j}\right)\right) x
\end{aligned}
$$

Sufficient stability conditions for closed-loop fuzzy systems (3) were derived in Theorem 1.

Theorem 1: If there exist symmetric and positive definite matrices $\mathrm{P}$ and $M_{i} \in R^{m \times n}$, the stability analysis of the robust fuzzy control is designed as follows:

$$
\begin{gathered}
A_{i} X+X A_{i}^{T}+M_{i}^{T} B_{i}^{T}+B_{i} M_{i}<? \\
A_{i} X+X A_{i}^{T}+A_{j} X+X A_{j}^{T}+M_{j}^{T} B_{i}^{T}+B_{i} M_{j}+ \\
M_{i}^{T} B_{j}^{T}+B_{j} M_{i} \geq 0
\end{gathered}
$$

where $X=P^{-1}, M_{i}=K_{i} X$.

Then, we got $K_{i}=M_{i} X^{-1}$

The state feedback law is constructed utilizing the resulting

$$
\left(K_{i}, i=1, \ldots, r\right)
$$

Since the state variables are not all available for measurement, a two stage design procedure is used to decouple the controller design from the observer design [17]. As indicated in [6], the fuzzy sliding mode observer allows a robust state estimation of the anaerobic digestion subject to unknown inputs.

\section{ANAEROBIC DIGESTION SYSTEM DESCRIPTION}

Anaerobic digestion is a biological multi-step process in which, organisms use available organic matter to support biological activity [2]. Organic solids are degraded and converted to methane $\mathrm{CH}_{4}$ and carbon dioxide $\mathrm{CO}_{2}$ in the absence of oxygen, commonly referred to collectively as biogas and treat domestic wastewater so that it can be safely reused as "recycled water". The organic solids consist of proteins, carbohydrates, and lipids, which are converted to biogas through four sequential, metabolic stages Fig. 1:

1) Hydrolysis, where complex organic molecules are broken down into simple sugars, amino acids, and fatty acids with the addition of hydroxyl groups,

2) Acidogenesis, where a further breakdown by acidogens into simpler molecules, volatile fatty acids (VFAs) occurs, producing ammonia, carbon dioxide and hydrogen sulfide as byproducts,

3) Acetogenesis, where the simple molecules from acidogenesis are further digested by acetogens to produce carbon dioxide, hydrogen mainly acetic acid and

4) Methanogenesis, where methane, carbon dioxide and water are produced by methanogens. There are several models available for the simulation of the anaerobic digestion of wastewater solids and sludges. Some of these models focus on a specific component of anaerobic digestion, such as microbial kinetics, while others attempt to encompass the overall digestion process [3], [17].

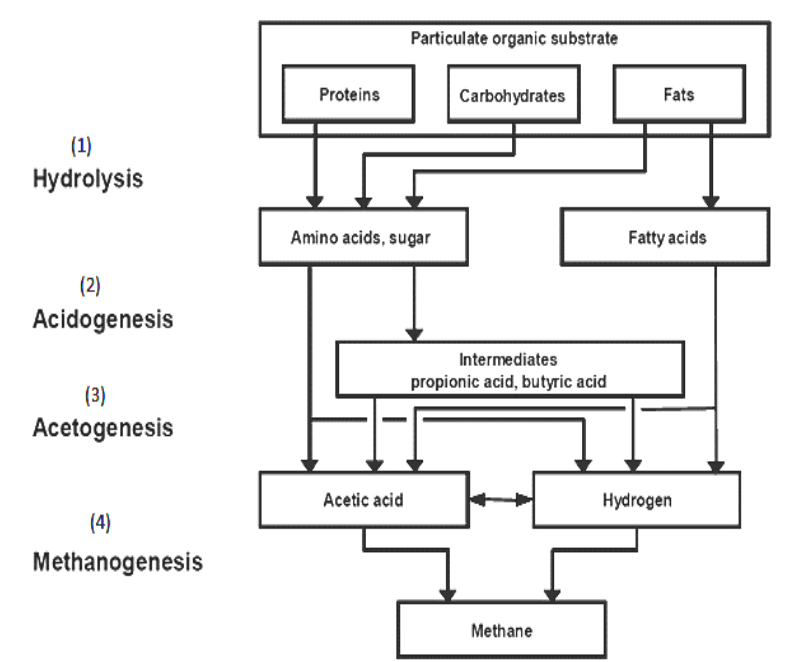

Fig. 1. The general schematic diagram of anaerobic digestion process.

$$
\left\{\begin{array}{l}
\dot{X}_{1}=\left(\mu_{1}-\alpha D\right) X_{1} \\
\dot{X}_{2}=\left(\mu_{2}-\alpha D\right) X_{2} \\
\dot{S}_{1}=D\left(S_{1}^{i n}-S_{1}\right)-k_{1} \mu_{1} X_{1} \\
\dot{S}_{2}=D\left(S_{2}^{i n}-S_{2}\right)-k_{2} \mu_{1} X_{1}-k_{3} \mu_{2} X_{2} \\
\dot{Z}=D\left(Z^{i n}-Z\right) \\
\dot{C}_{T I}=D\left(C_{T I}^{i n}-C_{T I}\right)+k_{7}\left(k_{8} P_{c o_{2}}+Z-C_{T I}-S_{2}\right)+ \\
k_{4} \mu_{1} X_{1}+k_{5} \mu_{2} X_{2} \\
Q_{C H_{4}}=k_{6} \mu_{2} X_{2}
\end{array}\right.
$$

where $\left[X_{1}, X_{2}, S_{1}, S_{2}, Z, C_{T I}\right]$ are respectively, the bacteria concentration acidogenic $(\mathrm{g} / \mathrm{L})$, methanogenic bacteria $(\mathrm{g} / \mathrm{L})$, soluble DCO $(\mathrm{gDCO} / \mathrm{L})$, total volatile fatty acids VFA $(\mathrm{mmol} / \mathrm{L})$, total inorganic carbon and total alkalinity (meq/L). For these variables "in" indicates the influent concentration. $D$ is the dilution rate and $Q_{\mathrm{CH}_{4}}$ is the biogas (methane) flow rate $(\mathrm{L} / \mathrm{h})$.

Nonlinear functions $\mu_{1}$ and $\mu_{2}$ represent the Haldane growth rates and have the following structure:

$$
\mu_{1}=\mu_{\max 1} \frac{S_{1}}{k_{s_{1}}+S_{1}} \text { and } \mu_{2}=\mu_{0} \frac{S_{2}}{k_{s_{2}}+S_{2}+\left(\frac{S_{2}}{k_{12}}\right)^{2}}
$$

where $k_{1}, \ldots, k_{8}$ are the yield coefficients.

The partial pressure $P_{\mathrm{CO}_{2}}$ of $\mathrm{CO}_{2}$ have the following structure:

$$
P_{\mathrm{CO}_{2}}=\frac{\Phi-\sqrt{\Phi^{2}-4 k_{8} P_{r}\left[\mathrm{CO}_{2}\right]}}{2 k_{8}}
$$

where $P_{r}$ is total pressure in the reactor (atm).

$$
\Phi=k_{8} P_{r}+\left[\mathrm{CO}_{2}\right]+\frac{k_{6} \mu_{2} X_{2}}{k_{1}} \text { and }\left[\mathrm{CO}_{2}\right]=C_{T I}+S_{2}-Z
$$

\section{Simulation AND RESUlts}

In this work, data sequences of the process inputs and 
biogas output evolution over 40 days are shown in Fig. 2.

The dilution rate $D$ is the manipulated variable and the unknown input vector is $d=\left[S_{1}^{i n}, S_{2}^{i n}, C_{T I}^{i n}, Z^{i n}\right]^{T}$, and the output vector is $y=\left[S_{1}, S_{2}, Z, Q_{C_{4}}\right]^{T}$.
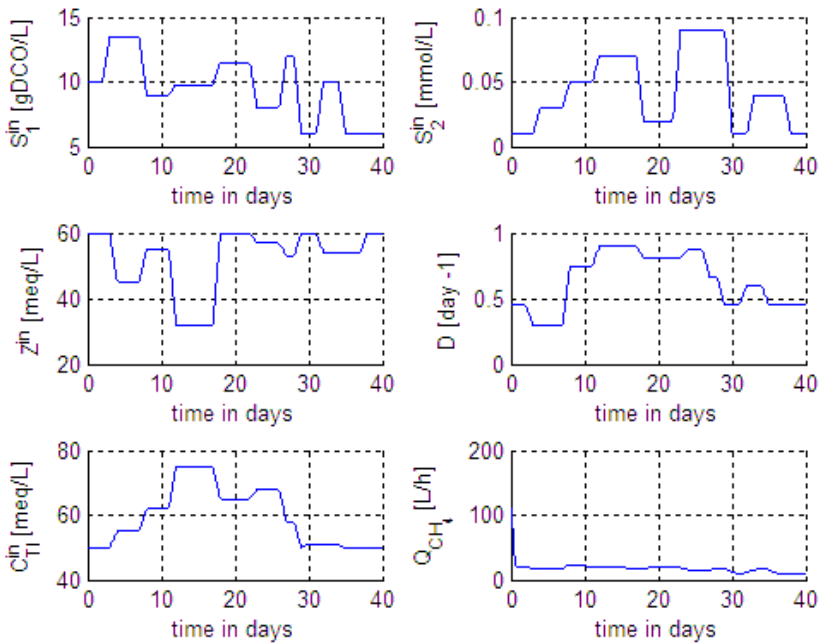

Fig. 2. Data sequences of the process inputs and biogas output.
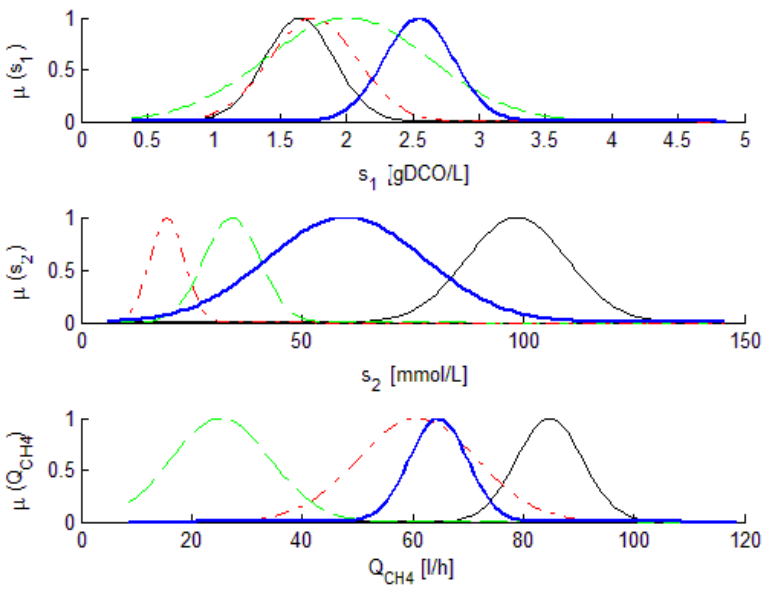

Fig. 3. Membership fonctions for $S_{1}, S_{2}$ and $Q_{\mathrm{CH}_{4}}$

T-S fuzzy model is constructed for an anaerobic digestion system using only four state space local models and without loss of generality, and by taking as premise variables $\xi=\left[S_{1}, S_{2}, Q_{\mathrm{CH}_{4}}\right]^{T}$. Fig. 3 shows the corresponding membership functions founded by using the GK algorithm.
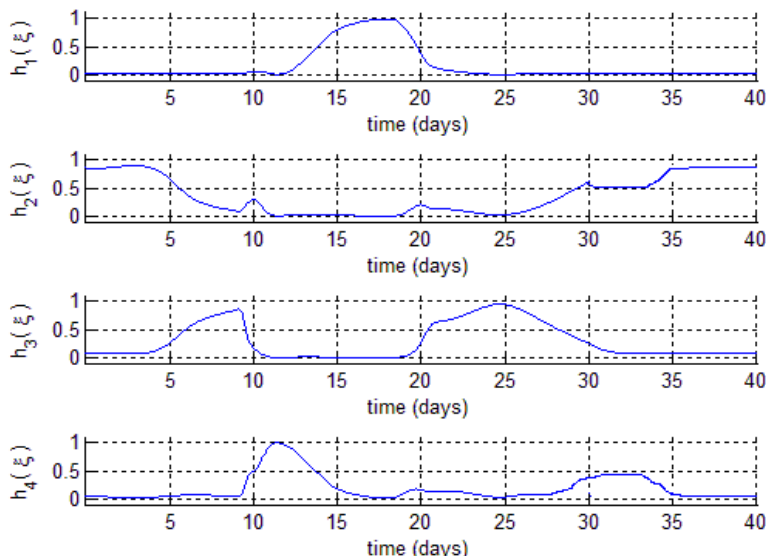

Fig. 4. The local model validity functions for the simulation interval.
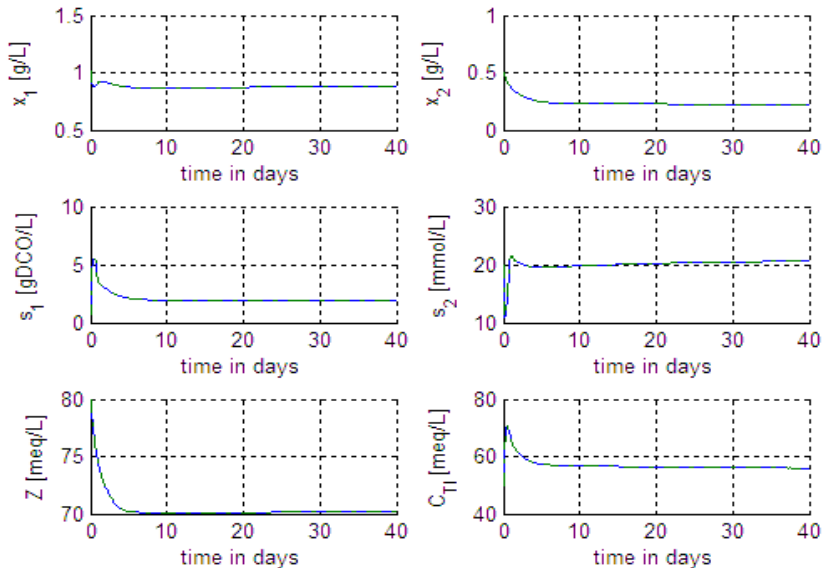

Fig. 5. Closed-loop stabilization with fuzzy controller.

Fig. 4 gives the local model validity functions over 40 days. By taking a vector of unknown inputs equal to zero, all the state variables tend to zero. Fig. 5 shows the results for a typical vector of unknown inputs different from zero that corresponds to a realistic influent water characteristics, and it can be said that the controller asymptotically stabilize the closed-loop of T-S fuzzy system.
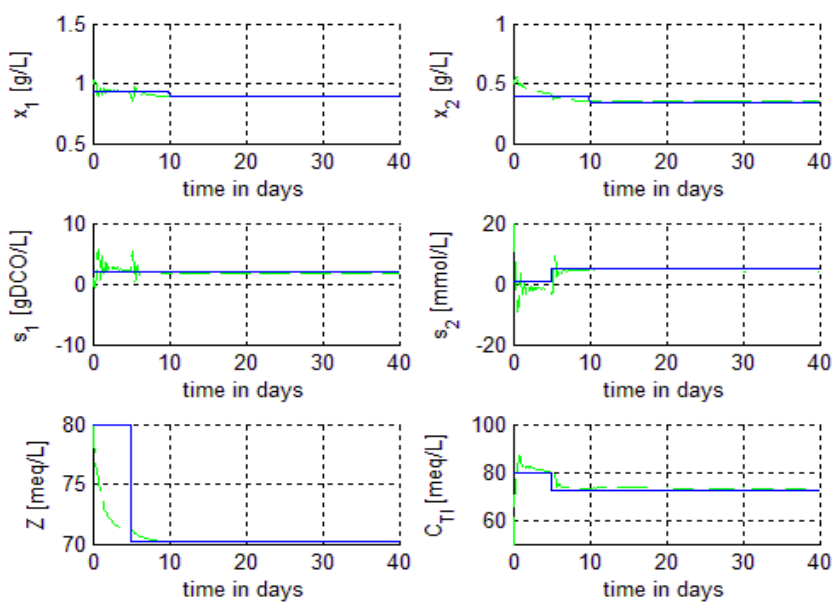

Fig. 6. Closed-loop results for an arbitrary state vector reference vector.

Fig. 6 illustrates the effectiveness of state feed-back tracking control for anaerobic digestion process by putting $\left(x(t)-x_{r}(t)\right)$ instead of $x(t)$ in (9), with $x_{r}(t)$ is the reference vector. The design of fuzzy robust tracking controller [18]-[22] will be treated in more details in our future work.

\section{CONCLUSION}

This paper deals with the design of a fuzzy controller for dynamic Takagi-Sugeno (TS) models for an anaerobic digestion process. First, the T-S fuzzy model is used to approximate the underlying nonlinear process dynamics. Then, a fuzzy state feedback controller is designed to ensure the stabilization of the closed-loop system by solving linear matrix inequalities LMIs problem, using an efficient numerical methods.

The simulation results prove the effectiveness of the proposed fuzzy controller (FC) for the anaerobic process subject to unknown inputs. In future work, the FC will be designed for output tracking and by taking into account 
parametric uncertainties.

\section{REFERENCES}

[1] R. Babuska, Fuzzy Modeling for Control, Amsterdam: Kluwer Publ. Co, 1998.

[2] T. Takagi and M. Sugeno, "Fuzzy identification of systems and its applications to modeling and control," IEEE Trans. Syst., Man, Cybern., vol. 15, pp. 116-132, Jan 1985.

[3] D. Theilliola, J. C. Ponsart, J. Harmand, C. Join, and P. Gras, "On-line estimation of unmeasured inputs for anaerobic wastewater treatment processes," Control Engineering Practice, vol. 11, pp. 1007-1019, 2003.

[4] G. Bastin and D. Dochain, On-Line Estimation and Adaptive Control of Bioreactors, Amsterdam: Elsevier, 1990.

[5] S. Carlos-Hernandez, J. F. Beteau, and E. N. Sanchez, "Design and real-time implementation of TS fuzzy observer for anaerobic wastewater treatment plants," in Proc. International Symposium on Intelligent Control, 2006, pp. 1252-1257.

[6] M. Boughamsa and M. Ramdani, "Design of fuzzy sliding mode observers for anaerobic digestion process," Engineering and Technology, vol. 3, pp. 117-122, 2013.

[7] F. Khaber, K. Zehar, and A. Hamzaoui, "State feedback controller design via Takagi-Sugeno fuzzy model: LMI approach,” Engineering and Technology, vol. 18, pp. 1158-1163, 2008.

[8] K. Tanaka, T. Ikeda, and H. O. Wang, "Fuzzy regulators and fuzzy obdervers: relaxed stability conditions and LMI-based designs," Transactions on Fuzzy Systems, vol. 6, no. 2, 1998.

[9] K. Tanaka and H. O. Wang, "Fuzzy regulators and fuzzy observers: A linear matrix inequality approach," in Proc. Conference on Decision and Control, 1997, pp. 1315-1320.

[10] T. Agustinah, A. Jazidie, M. Nuh, and H. Du, "Fuzzy tracking control design using observer-based stabilizing compensator for nonlinear systems," in Proc. International Conference on System Science and Engineering, 2010, pp. 275-280.

[11] J. Park, J. Kim, and D. Park, "LMI-based design of stabilizing fuzzy controllers for nonlinear systems described by Takagi-Sugeno fuzzy model," Fuzzy Sets and Systems, vol. 122, pp. 73-82, 2001.

[12] H. K. Lam, F. H. F. Leung, and P. K. S. Tam, "Nonlinear state feedback controller for nonlinear systems: stability analysis and design based on fuzzy plant model," IEEE Transaction on Fuzzy Systems, vol. 9, no. 4, pp. 657-661, 2001.

[13] M. Seidi et al., Fuzzy Control Systems: LMI-Based Design, 2012, ch. 18 , pp. 441-464.

[14] K. Tanaka and H. O. Wang, Fuzzy Control Systems Design and Analysis: A Linear Matrix Inequality Approach, John Wiley and Sons, Inc., 2001.

[15] M. Chadli, D. Maquin, and J. Ragot, "On the stability of multiple model systems," in Prco. European Control Conference, 2001, pp. 1894-1899.
[16] D. E. Gustafson and W. C. Kessel, "Fuzzy clustering with a fuzzy covariance matrix," in Proc. IEEE CDC, San Diago, CA, 1979, pp. 761-766.

[17] O. Bernard, Z. Hadj-Sadok, D. Dochain, A. Genovesi, and J. P. Steyer, "Dynamical model development and parameter identification for an anaerobic wastewater treatment process," Biotechnology and Bioengineering, vol. 75, no. 4, pp. 424-438, 2001.

[18] S. C. Tong, T. Wang, and H. X. Li, "Fuzzy robust tracking control for uncertain nonlinear," International Journal of Approximate Reasoning, vol. 30, no. 2, pp 73-90, 2002.

[19] C. S. Tseng, B. S. Chen, and H. J. Uang, "Fuzzy tracking control design for nonlinear dynamic system via T-S fuzzy model," IEEE Transactions on Fuzzy Systems, vol. 9, no. 3, pp. 381-392, 2001.

[20] F. Zheng, Q. G Wang, and T. H. Lee, "Output tracking control of MIMO fuzzy nonlinear systems using variable structure control approach," IEEE Transactions on Fuzzy Systems, vol. 10, no. 6, pp. 686-697, 2002

[21] C. Lin, Q. G. Wang, and T. H. Lee, " $\mathrm{H}_{\infty}$ output tracking control for nonlinear systems via T-S fuzzy model approach," IEEE Transaction on Systems, Man, and Cybernetics. Part B, Cybernetics, vol. 36, no. 2, pp. 450-457, 2006

[22] K. Y. Lian and J. J. Liou, "Output tracking control for fuzzy systems via output feedback design," IEEE Transactions on Fuzzy Systems, vol. 14 , no. 5, pp. 628-639, 2006.

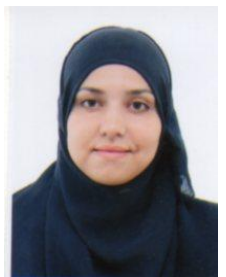

Mouna Boughamsa was born in 1987 in Annaba, Algeria. She received her master in 2009 in Automation from Badji-Mokhtar University Annaba, Algeria. She is currently a $\mathrm{PhD}$ student the Laboratory of Automatic and Signals Annaba (LASA), Badji-Mokhtar University, Department of Electronics, Annaba, Algeria. Her current research interests are bioprocesses, fuzzy systems, robust control and fault tolerant control.

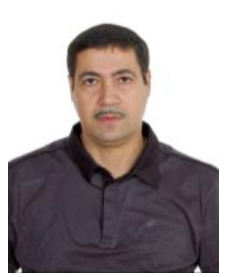

Messaoud Ramdani received the $\mathrm{PhD}$ degree in automatic control from the University of Annaba, Algeria, in 2006. He is currently a lecturer in the Department of Electronics, Faculty of Engineering, University Badji-Mokhtar of Annaba, Algeria. He has published over 36 journal and conference papers. His research interests include pattern recognition, fuzzy logic, machine learning, data mining and statistical process control. 